\title{
Fleischallergie durch Zeckenbiss?
}

\begin{abstract}
Allergische Reaktionen gegen Cetuximab können mit einer Überempfindlichkeit gegen rotes Fleisch zusammenhängen. Über die Hintergründe schreiben Scott Commins und Thomas Platts-Mills in dieser Ausgabe des Allergo Journal.
\end{abstract}

|rsprünglich waren Scott Commins (Chapel Hill) und Thomas PlattsMills (Charlottesville) auf der Suche nach den Ursachen von anaphylaktischen Reaktionen, die nach der Gabe des gegen den epidermalen Wachstumsfaktor-Rezeptor EGFR gerichteten monoklonalen Antikörper Cetuximab auftraten. Solche Reaktionen waren regional gehäuft in den Südstaaten der USA zu beobachten. Die beiden entdeckten, dass sich die IgE-vermittelten allergischen Prozesse nicht gegen ein Protein-, sondern gegen ein Kohlenhydratepitop richteten, und zwar gegen Galaktose-alpha-1,3-Galakotse (alpha-Gal). Ein solches Epitop sitzt auf der schweren Kette von Cetuximab. Interessanterweise trat die Anaphylaxie schon bei der ersten Gabe von Cetuximab auf. Die Sensibilisierung musste also eine andere Ursache haben als den monoklonalen Antikörper selbst.

Etwa um die gleiche Zeit, in den Jahren 2006 bis 2008, stießen Commins und Platts-Mills auf eine Gruppe von Patienten, die aus ungeklärter Ursache Episoden von generalisierter Urtikaria, Angioödemen und Anaphylaxie durchgemacht hatten - und zwar im selben geografischen Gebiet, in dem die Reaktionen auf Cetuximab beobachtet worden waren. Ein unmittelbarer Auslöser war nicht zu eruieren. Die Patienten ver- muteten aber, ihre Symptome könnten mit einer Fleischmahlzeit zusammenhängen, die sie einige Stunden zuvor zu sich genommen hatten.

Entsprechende kommerzielle Pricktests erbrachten keine eindeutigen Ergebnisse. Intradermale Tests und Pricktests mit frischem Extrakt aus rotem Fleisch zeigten aber stark positive Resultate. In Blutuntersuchungen waren spezifische IgE-Antikörper gegen Rind-, Schweine- und Lammfleisch, aber auch gegen alpha-Gal nachweisbar, was die Ergebnisse der Hauttests bestätigte.

Doch zwei Rätsel blieben einstweilen ungelöst: zum einen die geografische Häufung; und zum anderen der Umstand, dass die Reaktionen auf rotes Fleisch erst mit stundenlanger Verzögerung auftraten (im Gegensatz zu der sofortigen Reaktion auf Cetuximab und dem unmittelbaren Ansprechen im Hauttest).

Was die Geografie betraf, löste sich das Rätsel, als einer der Forscher, PlattsMills, bei sich selbst einen erheblichen Anstieg der Spiegel von alpha-Gal-IgE diagnostizierte. Zuvor war er wiederholt von Schildzecken der Art Amblyomma americanum gebissen worden. Deren Verbreitungsgebiet deckt sich mit den Regionen, in denen die Cetuximab- und die Fleischallergien aufgetreten waren. Auf Nachfrage gaben auch mindestens
$90 \%$ der betroffenen Patienten an, von Zecken gestochen worden zu sein, bevor sie ihre Fleischallergie entwickelten. Sensibilisierend wirkt vermutlich der Zeckenspeichel.

Die Verzögerung vom Fleischkonsum bis zur allergischen Reaktion erklären sich Commins und Platts-Mills mit der Zeit, die verstreichen muss, bis die allergene Form von alpha-Gal im Blut erscheint. Ob dies in Form eines Glykolipids geschieht, wie die Forscher annehmen, oder als Glykoprotein, ist erst noch zu klären.

Dr. Robert Bublak

Commins SP et al. The glycan did it: how the a-gal story rescued carbohydrates for allergists - a US perspective. Allergo J Int 2016; 25: 44-8

\section{Kommentar}

Die Arbeit von Scott Commins und Tom Platts-Mills ist die kurze und prägnante Zusammenfassung der „alpha-Gal-Story” aus der Feder der Erstbeschreiber. Ein absolutes Lesevergnügen und ein klassisches Beispiel wie Präzision in der klinischen Beobachtung kombiniert mit wissenschaftlicher Neugier und dem Mut zu lateralem Denken dazu führen kann, alte Dogmen über den Haufen zu werfen und wirklich Neues zu entdecken. Eine Pflichtlektüre für jeden Allergologen!

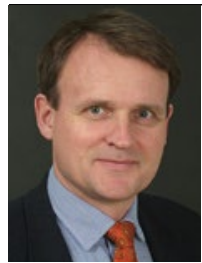

Prof. Dr. Thilo Jakob Direktor der Klinik für Dermatologie und Allergologie Universitätsklinikum Gießen u. Marburg, Standort Gießen

\section{Erhitzen nützt nicht viel bei Fleischallergie}

\section{Auch Stiche von Zecken, die wie der Gemeine Holzbock in Europa heimisch sind, können eine Fleischallergie auslösen. Die relevanten Allergene sind teils hitzestabil. Es nützt daher wenig, das Fleisch vor dem Verzehr gut durchzubraten oder zu kochen.}

$D_{\mathrm{t}}^{\mathrm{u}}$ urch das Kohlenhydratepitop Galaktose-alpha-1,3-Galakotse (alpha-Gal) vermittelte Allergien gegen rotes Fleisch sind auch in europäischen Ländern zu beobachten. Einen Überblick über die Situation in Schweden, wo inzwischen mehr als 200 solcher Fälle diagnostiziert worden sind, hat ein Wissenschaftlerteam um Marianne van Hage vom KarolinskaInstitut in Stockholm gegeben.

Die schwedischen Forscher fügen den vorhandenen Erkenntnissen zwei wesent- liche Einsichten hinzu. Zum einen stellen sie fest, dass wohl auch Ixodes ricinus, die als „Gemeiner Holzbock“ in Europa ansässige Zeckenart, ebenso wie seine Verwandten etwa in den USA und Australien, eine Fleischallergie auslösen kann. Bei so gut wie allen Patienten mit IgE-Antikörpern gegen alpha-Gal sind auch IgE-Antikörper gegen den Holzbock vorhanden. Dazu passt, dass alpha-Gal im Gastrointestinaltrakt von Ixodes ricinus nachgewiesen werden konnte. Für die Sensibilisierung gegen Fleisch sind Zeckenbisse anscheinend besonders wichtig. 